\title{
Small RNA metabolism in Arabidopsis
}

\author{
Vanitharani Ramachandran and Xuemei Chen \\ Department of Botany and Plant Sciences, University of California-Riverside, CA 92521, USA
}

\begin{abstract}
The Arabidopsis genome encodes two major classes of riboregulators ranging from 20 to 24 nucleotides in length: microRNAs (miRNAs) and small interfering RNAs (siRNAs). These small RNAs act as sequence-specific repressors of target gene expression either at the transcriptional level through DNA and/or histone methylation or at the posttranscriptional level through transcript cleavage or translational inhibition. Small RNAs are processed from precursor RNAs by one or more of the four DICER-LIKE (DCL) RNAse III enzymes. Small RNAs are then modified with a 2'-Omethyl group on their $3^{\prime}$ terminal ribose by HUA ENHANCER 1 (HEN1), a small RNA methyl transferase. In the absence of methylation, small RNAs become oligouridylated and/or degraded. The function of oligouridylation of small RNAs is currently unknown, but it is likely to cause small RNA degradation. Here, we review the biogenesis of small RNAs with an emphasis on the methylation and uridylation of small RNAs. We also discuss the major outstanding questions in small RNA metabolism.
\end{abstract}

\section{Introduction}

In eukaryotes small non-protein-coding RNAs of 20-30 nucleotides (nt) have emerged as key guide molecules to regulate various biological processes including developmental transition and patterning, responses to environment, maintaining genome stability, and defense agaist viruses and bacteria. One common theme underlying the actions of small RNAs is that an effector protein belonging to the argonaute (AGO) family binds a small RNA and confers the regulatory functions on target genes (reviewed in ${ }^{1}$ ). Argonaute proteins are classified into two phylogenetic subfamilies, the argonaute subfamily and the piwi subfamily. Whereas animals have members of both subfamilies, plant AGO proteins all belong to the argonaute subfamily. Concomitantly, plants appear to lack a class of RNAs known as piRNAs that are bound by piwi subfamily of argonaute proteins in animals (glossary).

microRNAs (glossary) were first identified through forward genetic screens as regulators of developmental timing in C. elegans 2,3 and later found to be nearly universally present in eukaryotes $3-8$. The fact that some miRNAs are conserved in sequence over long evolutionary distances 9,10 and the presence of miRNAs in the unicellular green alga Chlamydomonas reinhardtii 11,12 suggest that miRNA-based gene regulation is an ancient and evolutionarily conserved mechanism. miRNAs are processed from precursor RNAs that form hairpin structures by RNAse III enzymes (Drosha and Dicer in animals and DCL1 in plants) and are bound by an AGO subfamily member and inhibit their target genes by causing translational inhibition or RNA degradation (reviewed in ${ }^{13}$ ).

siRNAs (glossary) were first found in plants undergoing post-transcriptional gene silencing ${ }^{14}$ and later found to be universal effectors of RNA silencing in plants, fungi, and animals. However, siRNAs are not always directed towards transgenes or invading viruses. 
Large numbers of endogenous siRNAs have been discovered in C. elegans, rice and Arabidopsis ${ }^{15-19}$. In Arabidopsis, several distinct classes of endogenous siRNAs have been uncovered and found to regulate gene expression at the transcriptional and post-transcriptional levels. The regulatory functions of these endogenous siRNAs range from genome stability, responses to stress conditions, to developmental patterning.

\section{Major players in miRNA biogenesis and function}

The Arabidopsis genome contains hundreds of miRNA encoding regions designated as MIR genes. Unlike MIR loci in animals that tend to contain clusters of miRNAs and that are transcribed into polycistronic precursors (reviewed in ${ }^{20}$ ), plant $M I R$ genes are usually not clustered and each gene is transcribed into a precursor that gives rise to a single miRNA species. Like protein coding genes, $M I R$ genes are transcribed by RNA polymerase II (Pol II) and the primary transcripts (pri-miRNAs) are 5'-capped and 3'-polyadenylated ${ }^{21}$. A pri-miRNA contains a local hairpin structure in which the miRNA is embedded. The processes that generate the miRNAs from the pri-miRNAs are similar between plants and animals.

In animals, miRNAs are generated in two successive endonucleolytic steps (reviewed in ${ }^{20}$ ): an initial nuclear step converting pri-miRNAs into pre-miRNAs by Drosha and DiGeorge syndrome critical region gene 8 (DGCR8) protein, a double-stranded RNA (dsRNA) binding protein. This is followed by processing of pre-miRNAs into miRNA/miRNA* duplexes by Dicer in association with another dsRNA-binding protein, human immunodeficiency virus trans-activation responsive RNA-binding protein (TRBP) in the cytoplasm. In between, Exportin-5 transports the pre-miRNAs from the nucleus to the cytoplasm (reviewed in ${ }^{20}$ ).

In plants, miRNAs are generated from pri-miRNAs in a two- or sometimes multi-step process involving mainly DCL1 to yield miRNA-miRNA* duplexes $8,22,23$ (Figure 1). These processes are assisted by HYPONASTY LEAVES1 (HYL1), a dsRNA binding protein 24, 25. Mutations in either gene result in reduced accumulation of miRNAs and a concomitant increase in pri-miRNA levels ${ }^{26}$. DCL1 and HYL1 interact with each other in vitro and colocalize in the cell, suggesting that they form a complex to process pri-miRNAs ${ }^{26-29}$. SERRATE (SE), a zinc finger protein, interacts with DCL1 and HYL1 and also plays a role in the processing of pri-miRNAs to miRNAs since miRNAs are reduced in abundance while their pri-miRNAs accumulate to higher levels in serrate mutants $28,30,31$. After the miRNA/ miRNA* duplex is released from the pre-miRNA, the miRNA strand is selectively incorporated into the so-called RNA-induced silencing complex (RISC), whose major, if not only, protein component in Arabidopsis is ARGONAUTE 1 (AGO1) 32, 33 (Figure 1). AGO1 has endonucleolytic activity that cleaves the target mRNA in the middle of the mRNA-miRNA duplex 32,33 . HASTY, an Exportin-5 homolog, is thought to be involved in the transport of miRISCs or miRNA/miRNA* duplexes from the nucleus to the cytoplasm ${ }^{34}$ (Figure 1).

Although the biogenesis of the great majority of Arabidopsis miRNAs depends solely on DCL1 among the four DCLs, a few exceptions exist. miR822 and miR839 are DCL4- rather than DCL1-dependent ${ }^{35}$. This is likely due to the extensive sequence complementarity in the stemloop precursors, which makes these precursors better substrates for DCL4.

\section{Feed-back autoregulation}

miRNA biogenesis is under feedback regulation, a mechanism that helps ensure proper overall levels of miRNAs. The DCL1 gene encoding the predominant miRNA Dicer is itself a target of miR162. miR162-directed cleavage product of DCL1 mRNA is detected, indicating that $D C L 1$ expression is under negative regulation by miR $162^{36}$. In addition, one of the DCL1 introns hosts a miRNA, miR838 35 . This is a rare example where a MIR gene is located in the intron of a protein coding gene in Arabidopsis. Intronic miRNAs are very frequent in animals 
and the processing of these miRNAs does not appear to interefere with the splicing of the host $\mathrm{RNAs}^{37}$. In case of $D C L 1$, however, the processing of DCL1 pre-mRNA to release pre-miR838 probably generates two truncated transcripts that accumulate but that cannot give rise to DCL1 mRNA 35,36 . Therefore miR838 may also limit DCL1 expression. AGO1, which encodes a slicer that mediates the functions of miRNAs, is negatively regulated by miR $168^{38}$. miR403 targets $A G O 2$, however, the biological significance of $A G O 2$ in small RNA metabolism remains unknown.

\section{Major siRNA biogenesis pathways}

siRNAs are derived from long dsRNAs, which are generated through the transcription of inverted repeats or converted from single-stranded RNAs by RNA-dependent RNA polymerases (RDR). The dsRNAs are then processed into multiple siRNAs through the action of one or more of the four DCL proteins in Arabidopsis. Cloning followed by deep sequencing of small RNAs from Arabidopsis and rice show that numerous genomic loci give rise to siRNAs $16-18,39,40$. The biogenesis of three classes of endogenous siRNAs is best understood: RDR2-dependent siRNAs, trans-acting siRNAs (ta-siRNAs), and natural antisense siRNAs (nat-siRNAs).

RDR2-dependent siRNAs represent the largest and most diverse set of siRNAs in Arabidopsis. Deep sequencing of siRNAs from wild type and an $r d r 2$ mutant shows that these siRNAs can be mapped to loci throughout the five chromosomes but that pericentromeric regions have the highest density of distinct siRNAs as well as the highest number of siRNAs ${ }^{16}$. RDR2-dependent siRNAs are preferentially associated with transposon and retroelement loci and repetitive DNA, and tend to correspond to regions containing DNA methylation 16, 17, 40. At some loci, it has been shown that these siRNAs lead to transcriptional gene silencing by causing DNA methylation or histone $\mathrm{H} 3$ lysine 9 methylation 41,42 . The biogenesis of these siRNAs requires RDR $2^{42}$, which presumably converts a transcript from an siRNA generating locus into dsRNA. DCL3 then processes the dsRNA into $24 \mathrm{nt}$ siRNAs ${ }^{42}$. In the absence of DCL3, the other three DCLs can also process the dsRNAs into $21 \mathrm{nt}$ or $22 \mathrm{nt}$ siRNAs $16,39,42.24 \mathrm{nt}$ siRNAs are bound by AGO4, and perhaps AGO6, effectors that are required for DNA methylation and histone methylation at the target loci ${ }^{42-}$ 45 . The biogenesis of these siRNAs also requires one isoform of DNA-dependent RNA polymerase IV, pol IVa, and to some extent, another isoform pol IVb ${ }^{46-50}$. Pol IVa probably acts in the generation of dsRNA precursors to the siRNAs, while Pol IVb physically interacts with AGO4 and is thought to somehow facilitate siRNA-directed DNA or histone methylation $51-53$.

Trans-acting siRNAs (ta-siRNAs) are a class of endogenous siRNAs that act in trans, i.e. they regulate target genes that are different from their originating loci (reviewed in ${ }^{54}$ ). In Arabidopsis four TAS family genes (TAS1 to 4) encode non-protein coding transcripts that are themselves targets of miRNAs. TAS1 $(a, b$ and $c)$ and TAS2 are targets of miR173, TAS3 is targeted by miR390 and TAS4 is a target of miR834 $35,55-57$. The TAS transcripts are cleaved at specific positions by the corresponding miRISCs $35,55-57$. The cleavage fragments are probably stabilized by SGS3 and converted into dsRNAs by RDR6 56,57 . DCL4 then processes the dsRNAs into $21 \mathrm{nt}$ ta-siRNAs from one end of the dsRNA defined by the cleavage event $55,58-60$. Therefore, specific miRNA-programmed cleavage of the TAS transcripts triggers the production of ta-siRNAs and sets the registry for ta-siRNA production at $21 \mathrm{nt}$ increments from the cleavage site ${ }^{55}$. A dsRNA binding protein (DRB4) that is associated with DCL4, and SDE5, a putative homolog of a human mRNA export factor, also participate in tasiRNA biogenesis ${ }^{61-63}$. ta-siRNAs act through either AGO1 or AGO7 to regulate their target genes at the post-transcriptional level 32,61 . Notable ta-siRNA targets include $A R F 3$ and 
$A R F 4$, transcription factor genes that modulate auxin responses and plant development 55,61 , 64.

Nat-siRNAs are produced from loci with overlapping bidirectional transcripts. The two best studied nat-siRNAs are induced upon abiotic or biotic stresses and act in the stress response by down-regulating one of the gene pair that gives rise to the nat-siRNAs ${ }^{65,66}$. The biogenesis of the nat-siRNAs requires RDR6 and one or two of the DCL proteins.

\section{Subcellular locations of small RNA biogenesis}

miRNA processing may occur in subnuclear bodies. When fused to fluorescent proteins and expressed in Arabidopsis or N. benthamiana, DCL1 and HYL1 are co-localized in nuclear bodies, termed Dicing bodies or D-bodies, in addition to diffuse nucleoplasmic localization 27-29. The D-bodies do not co-localize with nucleolin, a nucleolus marker or Atcoilin, a marker for Cajal bodies, and are still present in an Atcoilin mutant lacking Cajal bodies, suggesting that D-bodies are not Cajal bodies $27-29$. D-bodies may also include SmD2 and SmD3 protiens that are also found in Cajal bodies 28 . Two pri-miRNAs have been found to co-localize with DCL1 and HYL1 in D-bodies, suggesting that D-bodies are miRNA processing centers or storage sites of the processing machinery or substrates 27,28 . The functional importance of D-bodies in miRNA biogenesis cannot be confirmed without the identification of mutations or conditions that disrupt D-bodies.

The processing of RDR2-dependent siRNAs may also occur in subnuclear bodies. A number of siRNAs and an siRNA precursor are concentrated in a subnucleolar body. A number of proteins in the biogenesis pathway, such as RDR2, DCL3, AGO4, and pol IVb, are co-localized with siRNAs in this body 52,53 . The co-localization of AGO4 with Cajal body markers identifies this site as the Cajal body ${ }^{52}$. It is thought that siRNA precursors are transported to Cajal bodies, where they are processed to siRNAs and where siRNAs are bound by AGO4 and pol IVb. The functional importance of Cajal bodies in siRNA processing and RISC assembly still awaits further interrogation of mutants lacking Cajal bodies.

\section{Small RNA modification by HEN1}

In plants, small RNA biogenesis requires an additional step apart from the endonucleolytic RNAse III cleavage steps. After miRNA/miRNA* or siRNA/siRNA* duplexes are released from their precursors by the DCL proteins, the duplexes are methylated on the $2^{\prime} \mathrm{OH}$ of the $3^{\prime}$ terminal ribose by HEN1, a small RNA methyltransferase ${ }^{67,68}$. In vitro biochemical studies using purified recombinant HEN1 protein show that HEN1 methylates miRNA and siRNA duplexes in a sequence-independent manner. HEN1 prefers 21 to 24 nt RNA duplexes with 2nt overhangs at their 3' ends, typical features of DICER products. miRNAs and siRNAs in wild-type plants but not hen 1 mutants are resistant to chemical reactions that target the $2^{\prime}$ and $3^{\prime} \mathrm{OH}$ in the $3^{\prime}$ terminal nucleotide, suggesting that at least one of the $\mathrm{OH}$ groups on the last ribose of small RNAs is modified in vivo ${ }^{68}$. Mass spectrometry analysis of affinity purified miR173 from plants revealed the presence of a single methyl group ${ }^{68}$. These studies demonstrate that small RNAs in Arabidopsis are present in $2^{\prime}-O$-methylated forms.

HEN1 homologs are present in various invertebrate and vertebrate animals ${ }^{8}$. Although animal miRNAs are not methylated, piRNAs are 2'-O-methylated by HEN1 homologs $69-71$.

\section{2'-O-methylation protects small RNAs from degradation and uridylation}

The protective role of the 2'-O-methyl group is recognized from analyses of miRNAs in hen 1 mutants that lack small RNA methylation ${ }^{72}$. In hen1 mutants, miRNAs fail to accumulate or accumulate to lower levels. When they are detectable by Northern blotting, miRNAs appear 
as a ladder of bands rather than as a single species ${ }^{72}$. miRNA cloning and sequencing revealed the presence of a number of $U$ residues at the $3^{\prime}$ end in hen 1 mutants, indicating that unmethylated miRNAs are prone to an unknown enzymatic activity that leads to oligouridylation ${ }^{72}$. Also, clones representing $3^{\prime}$-to-5' shorterning of miRNAs were found, implicating exonucleolytic degradation in the absence of methylation ${ }^{72}$. Therefore, methylation protects small RNAs from uridylation and degradation. siRNAs, such as tasiRNAs, RDR2-dependent siRNAs, and transgene-derived siRNAs all become heterogeneous in size in henl mutants, suggesting that oligouridylation also happens to siRNAs lacking methylation ${ }^{72}$.

Uridylation of RNAs has been found in other circumstances and has been correlated with RNA decay. The $5^{\prime}$ fragment of an mRNA cleaved by an miRISC becomes uridylated at its $3^{\prime}$ end and the uridylation is accompanied by $5^{\prime}$-to- $3^{\prime}$ degradation of the fragment ${ }^{73}$. In humans, histone mRNAs lack a polyA tail and their degradation is triggered by $3^{\prime}$ uridylation ${ }^{74}$. In case of miRNAs, it is possible that uridylation recruits an exonuclease to degrade the miRNAs.

\section{Outstanding questions in small RNA metabolism}

The last few years saw rapid progress in our understanding of small RNA metabolism in Arabidopsis such that the main framework of small RNA biogenesis is established. However, key outstanding questions remain to be addressed. First and the foremost is how small RNAs are turned over. Currently we know nothing about the stability of small RNAs or the enzymes that lead to their degradation. Uridylation and exonucleolytic degradation are implicated in miRNA degradation, but the enzymes catalyzing these reactions remain to be uncovered and the functions of these processes in miRNA metabolism await genetic confirmation by analysis of mutants in the corresponding genes. Another fundamental question is how some loci give rise to endogenous siRNAs while others do not. siRNAs tend to be associated with repetitive loci, but this is not true for all siRNAs. What are the defining features that predispose siRNA production? Finally, how small RNAs are loaded onto specific RISC complexes is completely unknown. There are $10 \mathrm{AGO}$ proteins in Arabidopsis and there is clear division of labor among the ones that have been studied. For example, AGO1 mediates the actions of miRNAs and transgene siRNAs, AGO4 and AGO6 mediate the functions of RDR2-dependent siRNAs, and AGO7 acts with ta-siRNAs from the TAS3 locus. However, how different small RNAs are channeled to different AGO proteins is unknown. Perhaps subcellular localization of small RNA biogenesis plays a role in the specificity of RISC loading. The functions of the remaining AGO proteins have yet to be elucidated.

\section{Acknowledgements}

Research in the Chen lab is supported by grants from the National Institutes of Health (GM61146) and National Science Foundation (MCB-0718029).

\section{Glossary}

Dicer

is an RNase III enzyme that cleaves double-stranded RNAs or single-stranded RNAs with imperfectly double-stranded hairpin regions to release 20-25 nt small RNAs

miRNAs

are 21-24 nt RNAs as the final products of non-protein coding genes. miRNAs are processed from longer, single-stranded precursor RNAs that form hairpin structures by Dicer and guide a silencing effector complex to target mRNAs through sequence complementarity 
SiRNAs

are 21-24 nt RNAs that are processed by Dicer from long double-stranded RNAs or single-stranded RNAs with extensive, perfect or near perfect hairpins. siRNAs can be derived from endogenous loci, transgenes, or viruses. Usually more than one siRNA species accumulate from a single precursor RNA. siRNAs guide a silencing effector complex to homologous DNA loci to trigger transcriptional gene silencing or to target mRNAs to cause transcript cleavage

\section{Piwi-interacting RNAs}

or piRNAs, are 25-30 nt RNAs found in the germline of invertebrate and vertebrate animals. They are generated in a Dicer-independent manner and act in transposon silencing and genome stability

\section{References}

1. Peters L, Meister G. Argonaute proteins: mediators of RNA silencing. Mol Cell 2007;26:611-623. [PubMed: 17560368]

2. Lee RC, et al. The C. elegans heterochronic gene lin-4 encodes small RNAs with antisense complementarity to lin-14. Cell 1993;75:843-854. [PubMed: 8252621]

3. Reinhart BJ, et al. The 21-nucleotide let-7 RNA regulates developmental timing in Caenorhabditis elegans. Nature 2000;403:901-906. [PubMed: 10706289]

4. Lagos-Quintana M, et al. Identification of novel genes coding for small expressed RNAs. Science 2001;294:853-858. [PubMed: 11679670]

5. Lau NC, et al. An abundant class of tiny RNAs with probable regulatory roles in Caenorhabditis elegans. Science 2001;294:858-862. [PubMed: 11679671]

6. Lee RC, Ambros V. An extensive class of small RNAs in Caenorhabditis elegans. Science 2001;294:862-864. [PubMed: 11679672]

7. Llave C, et al. Endogenous and silencing-associated small RNAs in plants. Plant Cell 2002;14:16051619. [PubMed: 12119378]

8. Park W, et al. CARPEL FACTORY, a Dicer homolog, and HEN1, a novel protein, act in microRNA metabolism in Arabidopsis thaliana. Curr Biol 2002;12:1484-1495. [PubMed: 12225663]

9. Axtell MJ, et al. Common functions for diverse small RNAs of land plants. Plant Cell 2007;19:17501769. [PubMed: 17601824]

10. Pasquinelli AE, et al. Conservation of the sequence and temporal expression of let-7 heterochronic regulatory RNA. Nature 2000;408:86-89. [PubMed: 11081512]

11. Molnar A, et al. miRNAs control gene expression in the single-cell alga Chlamydomonas reinhardtii. Nature 2007;447:1126-1129. [PubMed: 17538623]

12. Zhao T, et al. A complex system of small RNAs in the unicellular green alga Chlamydomonas reinhardtii. Genes Dev 2007;21:1190-1203. [PubMed: 17470535]

13. Bartel DP. MicroRNAs: genomics, biogenesis, mechanism, and function. Cell 2004;116:281-297. [PubMed: 14744438]

14. Hamilton AJ, Baulcombe DC. A species of small antisense RNA in posttranscriptional gene silencing in plants. Science 1999;286:950-952. [PubMed: 10542148]

15. Ambros V, et al. MicroRNAs and other tiny endogenous RNAs in C. elegans. Curr Biol 2003;13:807818. [PubMed: 12747828]

16. Kasschau KD, et al. Genome-wide profiling and analysis of Arabidopsis siRNAs. PLoS Biol 2007;5:e57. [PubMed: 17298187]

17. Lu C, et al. Elucidation of the small RNA component of the transcriptome. Science 2005;309:15671569. [PubMed: 16141074]

18. Nobuta K, et al. An expression atlas of rice mRNAs and small RNAs. Nat Biotechnol 2007;25:473477. [PubMed: 17351617]

19. Ruby JG, et al. Large-scale sequencing reveals 21U-RNAs and additional microRNAs and endogenous siRNAs in C. elegans. Cell 2006;127:1193-1207. [PubMed: 17174894] 
20. Kim VN, Nam JW. Genomics of microRNA. Trends Genet 2006;22:165-173. [PubMed: 16446010]

21. Xie Z, et al. Expression of Arabidopsis miRNA genes. Plant Physiol 2005;138:2145-2154. [PubMed: 16040653]

22. Kurihara Y, Watanabe Y. Arabidopsis micro-RNA biogenesis through Dicer-like 1 protein functions. Proc Natl Acad Sci U S A 2004;101:12753-12758. [PubMed: 15314213]

23. Reinhart BJ, et al. MicroRNAs in plants. Genes Dev 2002;16:1616-1626. [PubMed: 12101121]

24. Han MH, et al. The Arabidopsis double-stranded RNA-binding protein HYL1 plays a role in microRNA-mediated gene regulation. Proc Natl Acad Sci U S A 2004;101:1093-1098. [PubMed: 14722360]

25. Vazquez $F$, et al. The nuclear dsRNA binding protein HYL1 is required for microRNA accumulation and plant development, but not posttranscriptional transgene silencing. Curr Biol 2004;14:346-351. [PubMed: 14972688]

26. Kurihara Y, et al. The interaction between DCL1 and HYL1 is important for efficient and precise processing of pri-miRNA in plant microRNA biogenesis. Rna 2006;12:206-212. [PubMed: 16428603]

27. Fang Y, Spector DL. Identification of nuclear dicing bodies containing proteins for microRNA biogenesis in living Arabidopsis plants. Curr Biol 2007;17:818-823. [PubMed: 17442570]

28. Fujioka Y, et al. Location of a possible miRNA processing site in $\mathrm{SmD} 3 / \mathrm{SmB}$ nuclear bodies in Arabidopsis. Plant Cell Physiol 2007;48:1243-1253. [PubMed: 17675322]

29. Song L, et al. Arabidopsis primary microRNA processing proteins HYL1 and DCL1 define a nuclear body distinct from the Cajal body. Proc Natl Acad Sci U S A 2007;104:5437-5442. [PubMed: 17369351]

30. Lobbes D, et al. SERRATE: a new player on the plant microRNA scene. EMBO Rep 2006;7:10521058. [PubMed: 16977334]

31. Yang L, et al. SERRATE is a novel nuclear regulator in primary microRNA processing in Arabidopsis. Plant J 2006;47:841-850. [PubMed: 16889646]

32. Baumberger N, Baulcombe DC. Arabidopsis ARGONAUTE1 is an RNA Slicer that selectively recruits microRNAs and short interfering RNAs. Proc Natl Acad Sci U S A 2005;102:11928-11933. [PubMed: 16081530]

33. Qi Y, et al. Biochemical specialization within Arabidopsis RNA silencing pathways. Mol Cell 2005;19:421-428. [PubMed: 16061187]

34. Park MY, et al. Nuclear processing and export of microRNAs in Arabidopsis. Proc Natl Acad Sci U S A 2005;102:3691-3696. [PubMed: 15738428]

35. Rajagopalan R, et al. A diverse and evolutionarily fluid set of microRNAs in Arabidopsis thaliana. Genes Dev 2006;20:3407-3425. [PubMed: 17182867]

36. Xie Z, et al. Negative feedback regulation of Dicer-Like1 in Arabidopsis by microRNA-guided mRNA degradation. Curr Biol 2003;13:784-789. [PubMed: 12725739]

37. Kim YK, Kim VN. Processing of intronic microRNAs. Embo J 2007;26:775-783. [PubMed: 17255951]

38. Vaucheret H, et al. AGO1 homeostasis entails coexpression of MIRI68 and AGO1 and preferential stabilization of miR168 by AGO1. Mol Cell 2006;22:129-136. [PubMed: 16600876]

39. Henderson IR, et al. Dissecting Arabidopsis thaliana DICER function in small RNA processing, gene silencing and DNA methylation patterning. Nat Genet 2006;38:721-725. [PubMed: 16699516]

40. Zhang X, et al. Role of RNA polymerase IV in plant small RNA metabolism. Proc Natl Acad Sci U S A 2007;104:4536-4541. [PubMed: 17360559]

41. Hamilton A, et al. Two classes of short interfering RNA in RNA silencing. Embo J 2002;21:46714679. [PubMed: 12198169]

42. Xie Z, et al. Genetic and functional diversification of small RNA pathways in plants. PLoS Biol 2004;2:E104. [PubMed: 15024409]

43. Qi Y, et al. Distinct catalytic and non-catalytic roles of ARGONAUTE4 in RNA-directed DNA methylation. Nature 2006;443:1008-1012. [PubMed: 16998468]

44. Zheng X, et al. Role of Arabidopsis AGO6 in siRNA accumulation, DNA methylation and transcriptional gene silencing. Embo J 2007;26:1691-1701. [PubMed: 17332757] 
45. Zilberman D, et al. ARGONAUTE4 control of locus-specific siRNA accumulation and DNA and histone methylation. Science 2003;299:716-719. [PubMed: 12522258]

46. Herr AJ, et al. RNA polymerase IV directs silencing of endogenous DNA. Science 2005;308:118120. [PubMed: 15692015]

47. Kanno T, et al. Atypical RNA polymerase subunits required for RNA-directed DNA methylation. Nat Genet 2005;37:761-765. [PubMed: 15924141]

48. Mosher RA, et al. polIVb influences RNA-directed DNA methylation independently of its role in siRNA biogenesis. PNAS. 2008in press

49. Onodera Y, et al. Plant nuclear RNA polymerase IV mediates siRNA and DNA methylationdependent heterochromatin formation. Cell 2005;120:613-622. [PubMed: 15766525]

50. Pontier D, et al. Reinforcement of silencing at transposons and highly repeated sequences requires the concerted action of two distinct RNA polymerases IV in Arabidopsis. Genes Dev 2005;19:2030 2040. [PubMed: 16140984]

51. El-Shami M, et al. Reiterated WG/GW motifs form functionally and evolutionarily conserved ARGONAUTE-binding platforms in RNAi-related components. Genes Dev 2007;21:2539-2544. [PubMed: 17938239]

52. Li CF, et al. An ARGONAUTE4-containing nuclear processing center colocalized with Cajal bodies in Arabidopsis thaliana. Cell 2006;126:93-106. [PubMed: 16839879]

53. Pontes $\mathrm{O}$, et al. The Arabidopsis chromatin-modifying nuclear siRNA pathway involves a nucleolar RNA processing center. Cell 2006;126:79-92. [PubMed: 16839878]

54. Vaucheret H. Post-transcriptional small RNA pathways in plants: mechanisms and regulations. Genes Dev 2006;20:759-771. [PubMed: 16600909]

55. Allen E, et al. microRNA-directed phasing during trans-acting siRNA biogenesis in plants. Cell 2005;121:207-221. [PubMed: 15851028]

56. Peragine A, et al. SGS3 and SGS2/SDE1/RDR6 are required for juvenile development and the production of trans-acting siRNAs in Arabidopsis. Genes Dev 2004;18:2368-2379. [PubMed: 15466488]

57. Vazquez F, et al. Endogenous trans-acting siRNAs regulate the accumulation of Arabidopsis mRNAs. Mol Cell 2004;16:69-79. [PubMed: 15469823]

58. Gasciolli V, et al. Partially redundant functions of Arabidopsis DICER-like enzymes and a role for DCL4 in producing trans-acting siRNAs. Curr Biol 2005;15:1494-1500. [PubMed: 16040244]

59. Xie Z, et al. DICER-LIKE 4 functions in trans-acting small interfering RNA biogenesis and vegetative phase change in Arabidopsis thaliana. Proc Natl Acad Sci U S A 2005;102:12984-12989. [PubMed: 16129836]

60. Yoshikawa M, et al. A pathway for the biogenesis of trans-acting siRNAs in Arabidopsis. Genes Dev 2005;19:2164-2175. [PubMed: 16131612]

61. Adenot X, et al. DRB4-dependent TAS3 trans-acting siRNAs control leaf morphology through AGO7. Curr Biol 2006;16:927-932. [PubMed: 16682354]

62. Hernandez-Pinzon I, et al. SDE5, the putative homologue of a human mRNA export factor, is required for transgene silencing and accumulation of trans-acting endogenous siRNA. Plant J 2007;50:140148. [PubMed: 17397509]

63. Nakazawa Y, et al. The dsRNA-binding protein DRB4 interacts with the Dicer-like protein DCL4 in vivo and functions in the trans-acting siRNA pathway. Plant Mol Biol 2007;63:777-785. [PubMed: 17221360]

64. Hunter C, et al. Trans-acting siRNA-mediated repression of ETTIN and ARF4 regulates heteroblasty in Arabidopsis. Development 2006;133:2973-2981. [PubMed: 16818444]

65. Borsani O, et al. Endogenous siRNAs derived from a pair of natural cis-antisense transcripts regulate salt tolerance in Arabidopsis. Cell 2005;123:1279-1291. [PubMed: 16377568]

66. Katiyar-Agarwal S, et al. A pathogen-inducible endogenous siRNA in plant immunity. Proc Natl Acad Sci U S A 2006;103:18002-18007. [PubMed: 17071740]

67. Yang Z, et al. HEN1 recognizes 21-24 nt small RNA duplexes and deposits a methyl group onto the $2^{\prime} \mathrm{OH}$ of the 3' terminal nucleotide. Nucleic Acids Res 2006;34:667-675. [PubMed: 16449203] 
68. Yu B, et al. Methylation as a crucial step in plant microRNA biogenesis. Science 2005;307:932-935. [PubMed: 15705854]

69. Horwich MD, et al. The Drosophila RNA methyltransferase, DmHen1, modifies germline piRNAs and single-stranded siRNAs in RISC. Curr Biol 2007;17:1265-1272. [PubMed: 17604629]

70. Kirino Y, Mourelatos Z. The mouse homolog of HEN1 is a potential methylase for Piwi-interacting RNAs. Rna 2007;13:1397-1401. [PubMed: 17652135]

71. Saito K, et al. Pimet, the Drosophila homolog of HEN1, mediates 2'-O-methylation of Piwiinteracting RNAs at their 3' ends. Genes Dev 2007;21:1603-1608. [PubMed: 17606638]

72. Li J, et al. Methylation protects miRNAs and siRNAs from a 3'-end uridylation activity in Arabidopsis. Curr Biol 2005;15:1501-1507. [PubMed: 16111943]

73. Shen B, Goodman HM. Uridine addition after microRNA-directed cleavage. Science 2004;306:997. [PubMed: 15528436]

74. Mullen TE, Marzluff WF. Degradation of histone mRNA requires oligouridylation followed by decapping and simultaneous degradation of the mRNA both $5^{\prime}$ to $3^{\prime}$ and $3^{\prime}$ to $5^{\prime}$. Genes Dev 2008;22:50-65. [PubMed: 18172165]

75. Mi, Shijun; Cai, Tao; Hu, Yugang; Chen, Yemiao; Hodges, Emily; Ni, Fangrui; Wu, Liang; Li, Shan; Zhou, Huanyu; Long, Chengzu; Chen, She; Hannon, Gregory J.; Qi, Yijun. Sorting of Small RNAs into Arabidopsis Argonaute Complexes Is Directed by the 5' Terminal Nucleotide. Cell 2008 2008;133:116-127.

76. Montgomery, Taiowa A.; Howell, Miya D.; Cuperus, Josh T.; Li, Dawei; Hansen, Jesse E.; Alexander, Amanda L.; Chapman, Elisabeth J.; Fahlgren, Noah; Allen, Edwards; Carrington, James C. Specificity of ARGONAUTE7-miR390 Interaction and Dual Functionality in TAS3 Trans-Acting siRNA Formation. Cell 2008 2008;133:128-141. 


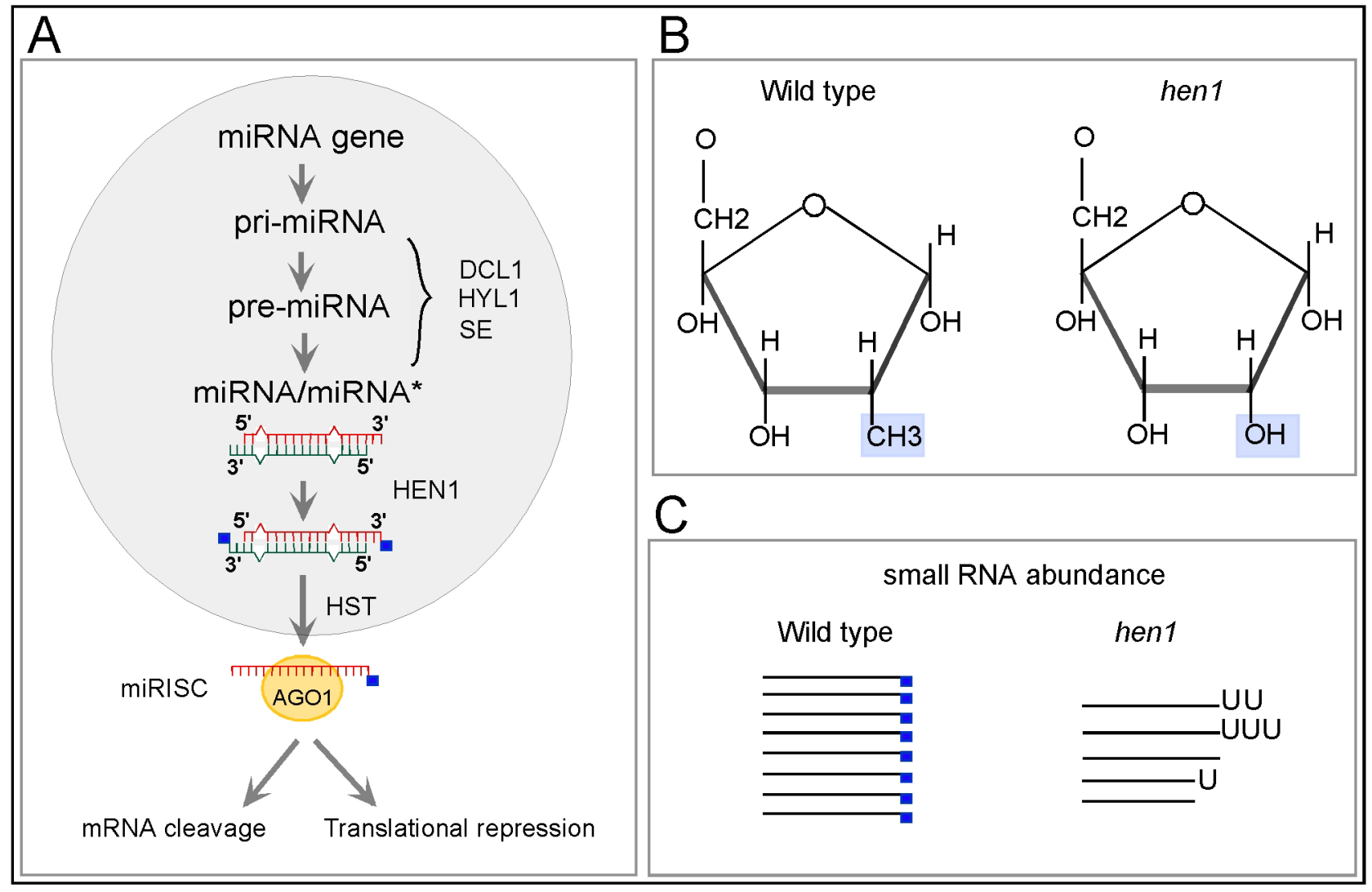

Figure 1.

Schematic illustration of miRNA metabolism in Arabidopsis. (A) Major steps in miRNA biogenesis. Primary miRNA transcripts (pri-miRNAs) are processed into precursor miRNAs (pre-miRNA) and then to miRNA/miRNA* duplexes by the coordinated activities of DCL1, HYL1 and SE within the nucleus. miRNA and miRNA* strands are represented in red and green, respectively. Both strands of the miRNA/miRNA* duplex are $2^{\prime}-O$-methylated on the $3^{\prime}$ terminal ribose by HEN1, a small RNA methyltransferase (The $2^{\prime}$ - $O$-methyl groups are represented by blue squares). The miRNA strand gets incorporated into an AGO1 containing RNA-induced silencing complex (miRISC) and directs either mRNA cleavage or translational inhibition. miRISCs or an intermediate, such as miRNA/miRNA* or methylated miRNA/ miRNA*, in the biogenesis pathway is exported from the nucleus (represented by the oval) to the cytoplasm by the Exportin-5 homolog HASTY. (B) The position of the methyl group in miRNAs. In wild-type plants, HEN1 adds a methyl group $\left(\mathrm{CH}_{3}\right.$, shaded box $)$ at the $2^{\prime}$ position of the last ribose. In hen 1 mutants, miRNAs are not methylated and have a $2^{\prime}$ hydroxyl group (shaded box). (C) A diagram depicting the abundance and modification of miRNAs in wildtype plants and in hen 1 mutants. The abundance and size of miRNAs are represented by the numbers and length of the lines, respectively. In wild-type plants, miRNAs are methylated and accumulate as full-length molecules. In hen 1 mutants, miRNAs are not methylated and accumulate at much lower levels as modified molecules. The modification includes $3^{\prime}$ truncation, $3^{\prime}$ uridylation, or $3^{\prime}$ truncation plus $3^{\prime}$ uridylation. 


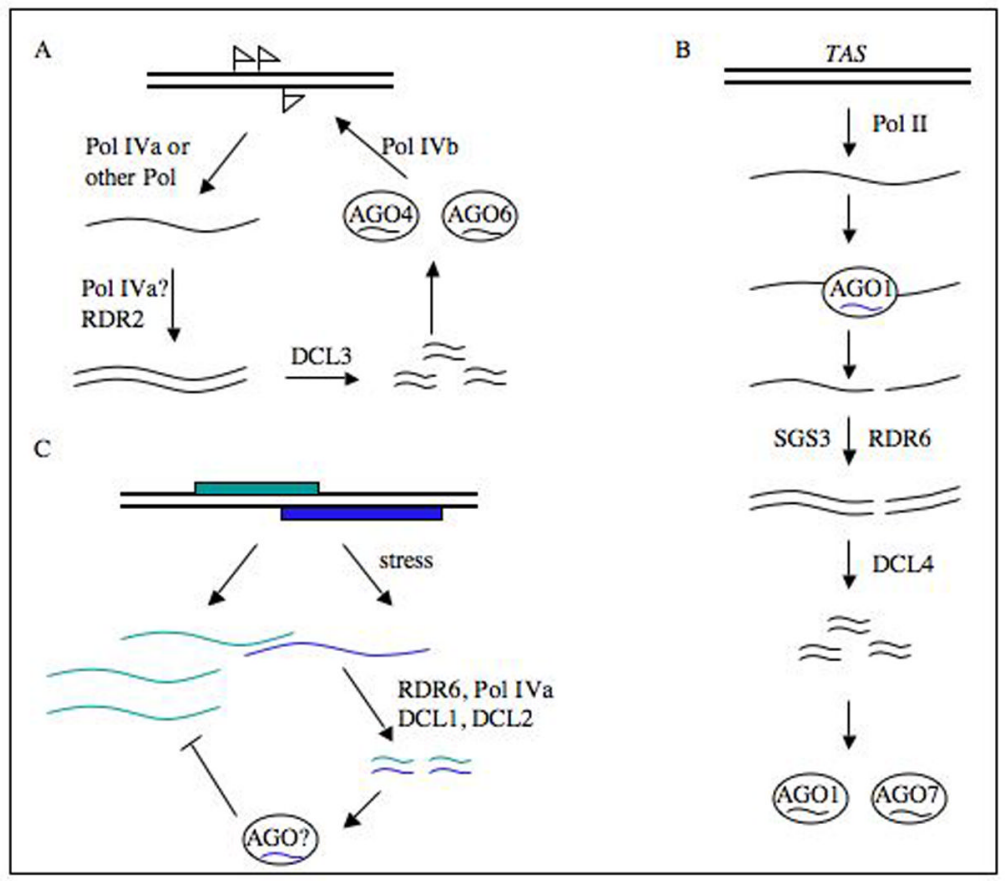

Figure 2. 